\title{
The neural correlates of visual self-recognition
}

\author{
Christel Devue ${ }^{1,2}$ and Serge Brédart ${ }^{1}$ \\ ${ }^{1}$ Centre des Neurosciences Cognitives et Comportementales, Université de Liège, Belgium \\ ${ }^{2}$ Cognitive Psychology, Vrije Universiteit Amsterdam, The Netherlands \\ In press in Consciousness and Cognition
}

Correspondence to:

Christel Devue

Department of Cognitive Science

Université de Liège

Bd du Rectorat, 5 (Bât. B32)

B-4000 Liège

Belgium

Email: cdevue@ulg.ac.be

Tel.: +32[0]4 3662021

Fax: $+32[0] 43662859$ 


\begin{abstract}
This paper presents a review of studies that were aimed at determining which brain regions are recruited during visual self-recognition, with a particular focus on self-face recognition. A complex bilateral network, involving frontal, parietal and occipital areas, appears to be associated with self-face recognition, with a particularly high implication of the right hemisphere. Results indicate that it remains difficult to determine which specific cognitive operation is reflected by each recruited brain area, in part due to the variability of used control stimuli and experimental tasks. A synthesis of the interpretations provided by previous studies is presented. The relevance of using self-recognition as an indicator of self-awareness is discussed. We argue that a major aim of future research in the field should be to identify more clearly the cognitive operations induced by the perception of the self-face, and search for dissociations between neural correlates and cognitive components.
\end{abstract}

\title{
Acknowledgements
}

C.D. is a Postdoctoral Researcher of the Belgian National Fund for Scientific Research (FRSFNRS). S.B. was funded by the French-speaking community of Belgium (ARC 06/11-340). 


\section{Introduction}

Our own face is an important component of our identity, besides other self-related information such as our own name, hometown, occupation, preferences and so on. However, contrary to other self-related information our face is a unique self-referential stimulus, presumably our most distinctive physical feature (Tsakiris, 2008). Indeed, our own face is a property that we do not share with other people (with the exception of twins) whereas it is quite common to share properties such as the occupation, the hometown or even the name with other people (Devue \& Brédart, 2008). During the last forty years, the ability of self-recognition in a mirror has been extensively investigated by researchers searching for signs of 'self-consciousness' or some sense of personal identity in infants and in animals (Amsterdam, 1972; Gallup, 1970; for a review see Keenan, Gallup, \& Falk, 2003a). The face is often seen as the emblem of the self (McNeill, 1998), and Cole (1999) has described in his book 'About face' how life of people suffering from problems touching their own face (e.g., facial paralysis or disfigurement) is deeply affected. More recently, with the occurrence of face transplants procedures, psychologists started to consider the possible deleterious effects of such surgical procedures on the patients' sense of identity (Bluhm $\&$ Clendenin, 2009). A much more mundane but significant example of the importance of the face in defining a person is illustrated by the fact that since the propagation of photography, passports or driving licenses from all over the world contain an identity picture in addition to the owner's name.

Over the last ten years, the interest towards visual self-recognition has grown among the neuroscientific community. One of the reasons is that a lot of researchers in that field have made the assumption that presenting people with stimuli depicting themselves is a clear and straightforward way to study the neural correlates of the self and of self-awareness. The present review addresses visual self-recognition and its neural correlates. More specifically, the issues of determining the hemispheric dominance of self-recognition (the lateralization issue) and determining which brain areas are consistently involved during self-recognition (the localization issue) will be examined here. Note that this topic is clearly much more restricted than "selfprocessing" in general. The important point of the relationship between visual self-recognition and self-awareness will be addressed in the discussion.

\section{The lateralization of visual self-recognition}

A number of studies compared reaction times for contralateral left or right hand use as a measure of hemispheric dominance of self-face recognition. People tend to respond faster to their own face than other familiar faces, either famous (Miyakoshi, Kawayama, Iidaka, \& Ohira, 2010; Tacikowski \& Nowicka, 2010) or friend's faces (Keyes \& Brady, 2010; Sui, Liu, \& Han, 2009; Sui, Zhu, \& Han, 2006; Sugiura, Sassa, Jeong, Horie, Sato, \& Kawashima, 2008) in explicit or incidental face identification tasks. However several studies showed that this speed advantage for the self-face only occurs when participants respond with their left hand (Keenan, McCutcheon, Freund, Gallup, Sanders, \& Pascual-Leone, 1999; Ma \& Han, 2010; Platek \& Gallup, 2002; Platek, Thomson, \& Gallup, 2004). Because of contralateral motor control, this finding was interpreted as reflecting right hemisphere dominance of self-face recognition. This left-hand advantage was demonstrated with another dependent measure using an innovative experimental procedure in which the participants' task was to stop a movie of a morphed face that transitioned between a famous face and self-face, or between a famous face and a co-worker's face, as soon as they thought that the image looked more like self, or the co-worker, than the famous person (Keenan, Freund, Hamilton, Ganis, \& Pascual-Leone, 2000; Keenan, Ganis, Freund, \& PascualLeone, 2000). In these studies participants stopped the "famous to self" movie sooner when 
responding with the left hand than when responding with the right hand. Such hand difference did not occur for the "famous to co-worker" movie. In other words, participants were more sensitive to self when they responded with their left hand than when they did it with the right hand.

Although the left-hand advantage seems to provide a first support for a preferential role of the right hemisphere in processing the self-face, other behavioral studies reported data supporting a left hemisphere bias for self-recognition. In these studies, participants were presented with composite faces of themselves and a friend. They were asked to choose which of two symmetric self-faces (one made from the left half and one made of the right half) looked more like themselves. They preferentially chose the composite made of the right half face, i.e. the half face that lies in their right visual field when they look at themselves in the mirror. When asked to choose which symmetric face was more representative of their friend, participants chose the composite made of the right half of their friend' face. This choice represents the opposite bias since that half face lies in their left visual field when they look at their friend (Brady, Campbell, \& Flaherty, 2004). These results suggest that the left hemisphere is dominant for self-recognition, and the right hemisphere is dominant for the recognition of other familiar faces.

The study of split-brain patients also provided important data for the assessment of brain lateralization of self-recognition. Sperry, Zaidel, \& Zaidel (1979) examined two split-brain patients and reported that both hemispheres were capable of self-recognition. More recently, several studies used the technique of presenting split-brain patients with varying levels of images of self and familiar faces being morphed together (Turk, Heatherton, Kelley, Funnell, Gazzaniga, \& Macrae, 2002), or levels of self and familiar faces each being morphed with another face (Uddin, Rayman, \& Zaidel, 2005b). Stimuli were presented laterally to each hemisphere. The patients' task was to choose whether a given image portrayed her/himself or a familiar person. Turk et al. (2002) found that the proportion of either self or other responses increased, until becoming excellent, as the images approached completeness (i.e. 100\% of self or familiar face in the morph). This finding suggests that both hemispheres are capable of self recognition and familiar face recognition. These authors also found that the patient's proportion of positive responses while he judged whether or not the image was self was significantly higher when the images were presented to the left than to the right hemisphere. The opposite bias was observed when the task was to determine whether the presented face was that of a familiar person. These results support the view that the left hemisphere plays a dominant role in self-recognition. Uddin et al. (2005b) also examined a split-brain patient's performances using a similar procedure but they obtained quite different results. They also found that both hemispheres are capable of selfrecognition. Their results, contrary to those reported by Turk et al. (2002), showed no indication of a hemispheric specialization for self-recognition. Moreover, this patient was able to recognize the familiar face with her right hemisphere only. The same kind of task of self/other judgment from morphs was also used with another split brain patient (Keenan, Wheeler, Platek, Lardi, \& Lassonde, 2003). In that study, stimuli were presented centrally and the patient carried out the self-search and the familiar other search task with either the left or the right hand. In the selfsearch condition, the proportion of correct identifications was higher and the proportion of false positives was lower when responding with left hand than with the right hand. No hand advantage occurred when searching for the familiar face. These results are consistent with the hypothesis of right hemisphere dominance of self-recognition. Keenan and colleagues drew the same conclusion from another study in which patients with intractable epilepsy underwent an intracarotid amobarbital procedure (Wada test) consisting in anaesthetizing one cerebral hemisphere at a time (Keenan, Nelson, O'Connor, \& Pascual-Leone, A., 2001). During anaesthesia, five patients were presented with a picture of their own face morphed with a celebrity's face (e.g. Marilyn Monroe). After they recovered from anaesthetization, patients were given a force-choice task in which they 
had to choose the picture they had been shown earlier. After anaesthetization of the right hemisphere patients were more likely to choose the famous face. By contrast, when the left hemisphere had been anaesthetized (and the right hemisphere was still active), all patients chose their own face.

Hemispheric biases for self-face recognition have also been studied with repeated transcranial magnetic stimulation (rTMS). This technique allows the creation of virtual lesions in chosen regions and an assessment of their effects. Uddin, Molnar-Szakacs, Zaidel and Iacobini (2006) demonstrated an implication of the right (but not left) inferior parietal lobule during selfface recognition. Indeed, an inhibition of this region decreased the sensitivity of participants to detect their own face among morphed images of themselves and another highly familiar person. More recently, Heinisch, Dinse, Tegenthoff, Juckel, and Brüne (2010) showed that the right temporo-parietal junction was important in self-other distinction and that the prefrontal structures are involved in self-evaluation.

Further evidence of a crucial intervention of the right hemisphere in self-face recognition comes from studies of patients with a deficit which involves misidentification of one's own mirror reflection as another person. Extensive right hemisphere damage is usually observed in patients suffering from such mirrored-self misidentification (Breen, Caine, \& Coltheart, 2001; Feinberg \& Keenan, 2005).

A conclusion that is obvious from studies reported in the present section is that hemispheric dominance for self-face recognition has been a matter of controversy. However an important result reported in all studies of split-brain patients is that both cerebral hemispheres are capable of self-face recognition (Keenan et al., 2003b; Sperry et al., 1979; Turk et al., 2002; Uddin et al., 2005b). However, as far as hemispheric dominance of self-face recognition is concerned, the conclusions of these studies are contradictory: Turk et al. (2002) concluded that the left hemisphere is dominant, Keenan et al. (2003b) claimed that the right hemisphere is dominant, and Uddin et al. (2005b) found no hemispheric dominance. Besides these studies of split-brain patients, the observation of a left-hand advantage observed in self-recognition tasks only, the results from rTMS studies, as well as the preferential interpretation of a morph being self-face after anaesthetization of the left but not the right hemisphere support the hypothesis of right hemisphere dominance. By contrast, results from Brady et al.'s (2004) experiments with composite faces are more consistent with a left hemisphere hypothesis. In the next section, we will address the issue of which brain areas, in the left or the right hemisphere, are selectively recruited in self-face recognition.

\section{Neuroimaging studies}

A summary of the main characteristics of the methods used in the studies reviewed here can be found in Table 1.

In previous studies, the cerebral regions involved during self-face recognition have been compared to those involved during the processing of faces varying in familiarity, namely from unfamiliar faces, to famous or personally familiar faces (i.e. co-worker, friend, partner, mother, sibling). The studies using familiar faces as control offer a better guarantee that activity found with the self-face is due to its self-relevance and not to its familiarity. Therefore we present results obtained with familiar and unfamiliar control faces separately. Figure 1 shows the percentage of activation reported in the different lobes in all studies comparing the neural correlates of the selfface to that of another face. When the same region within a lobe was activated several times using different contrasts in the same study (e.g. when different types of visual material such as pictures and movies or different types of tasks such as passive or active viewing were used), we only counted it as one "hit" on condition that the various contrasts included the same category of face 
of comparison (i.e. familiar versus unfamiliar). In other words, if the same region was found in a contrast comparing the self-face to another familiar face and another comparing the self-face to an unfamiliar face in the same study, the number of "hits" for that region would equal 2 in the first panel of that figure. Figure $1 \mathrm{~b}$ is based on peaks of activation reported during the processing of the self-face by comparison with other highly familiar faces (famous or personally familiar) and Figure $1 \mathrm{c}$ on regions activated when the self-face processing was compared with that of unfamiliar faces.

Table 1. Summary of methods used in previous neuroimaging studies.
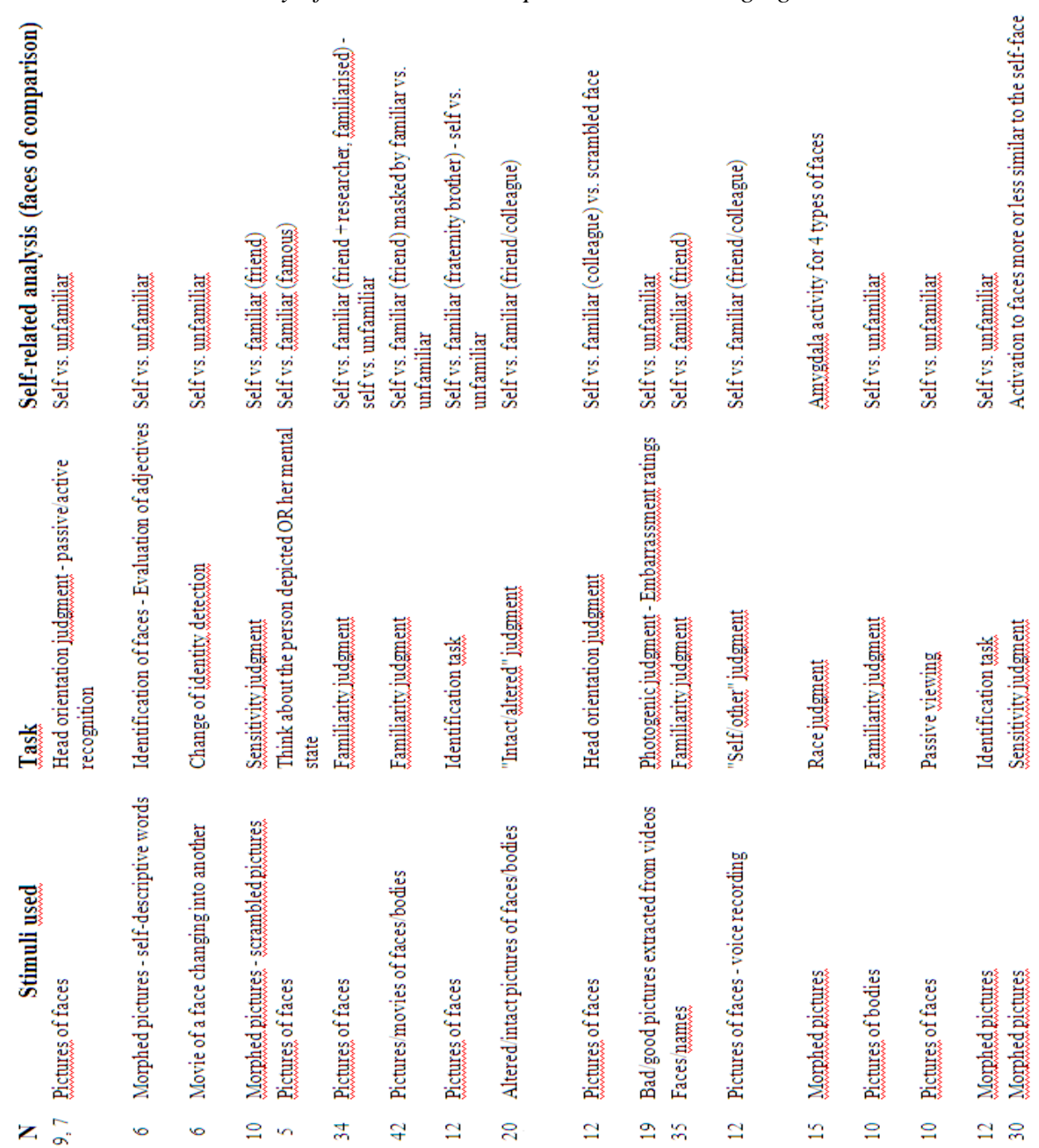

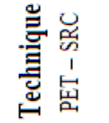

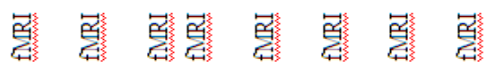
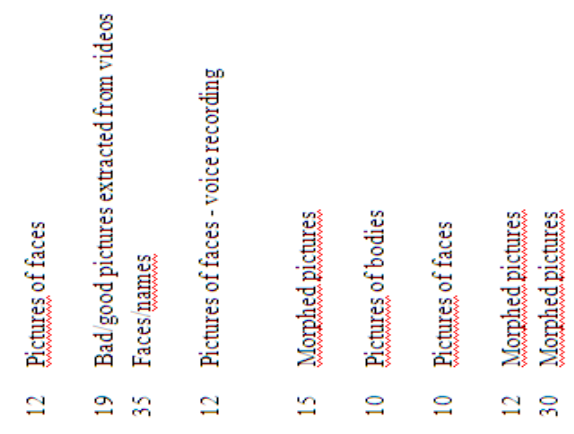

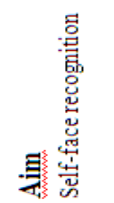
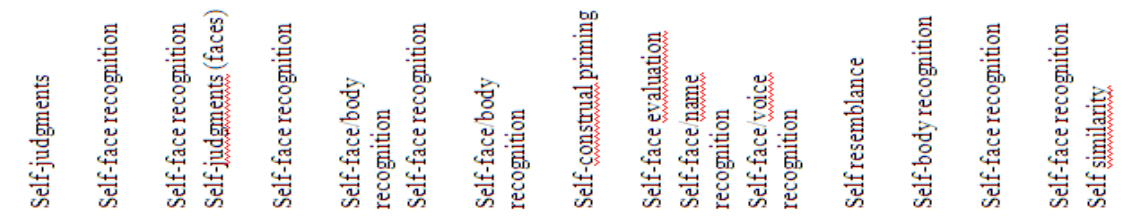

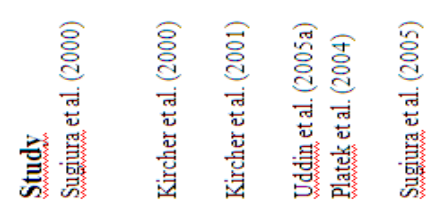
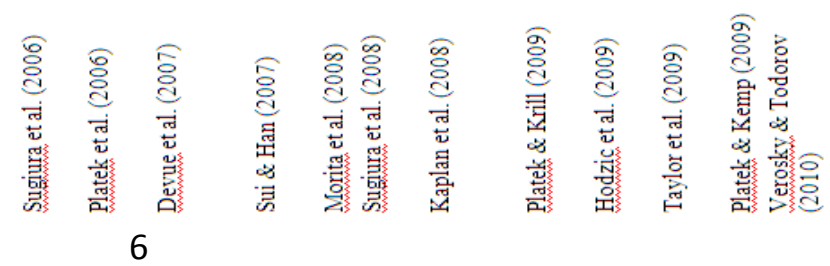
Figure 1. Percentage of activation reported in cerebral areas in each hemipshere on all activations reported in previous neuroimaging studies: (a) all studies comparing self-face processing to another face processing collapsed;

(b) in studies comparing self-face processing to familiar face processing; (c) in studies comparing self-face processing to unfamiliar face processing.

Self-face vs. other faces (overall)

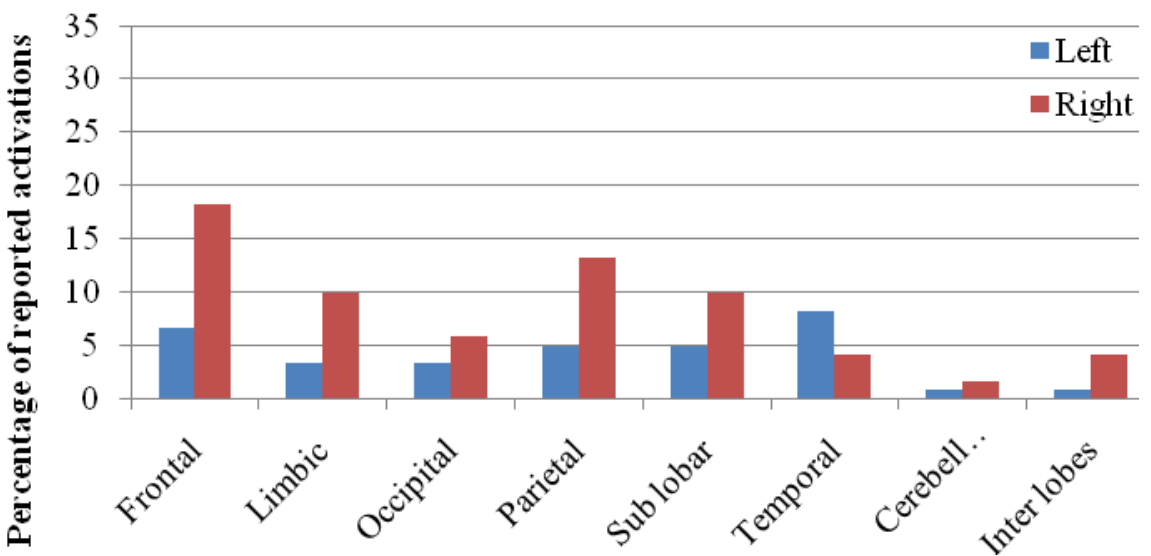

$\mathbf{a}$

Self-face vs. familiar faces

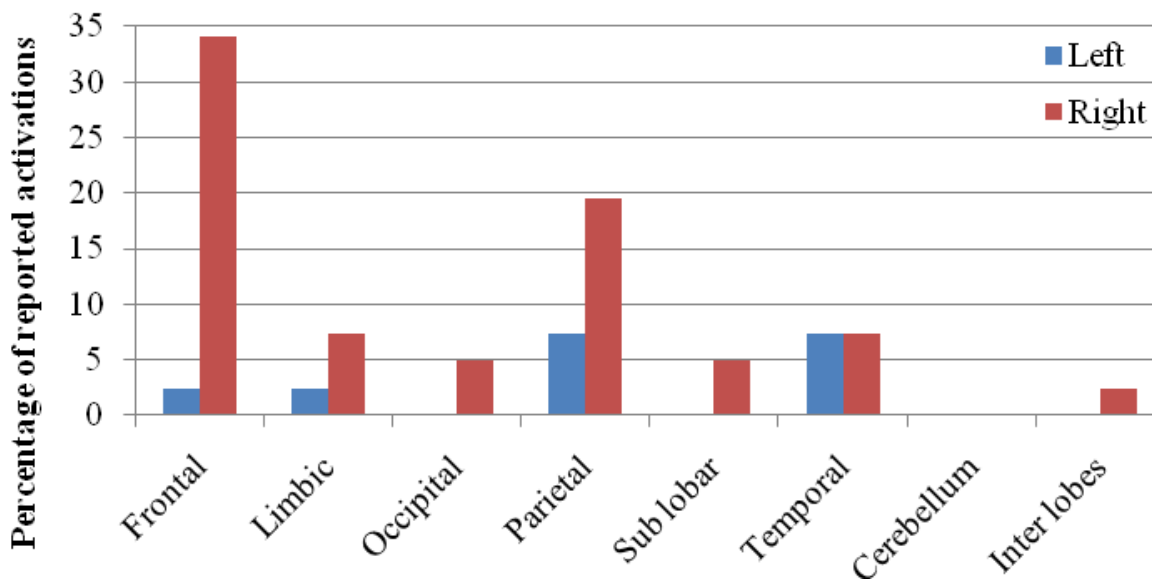




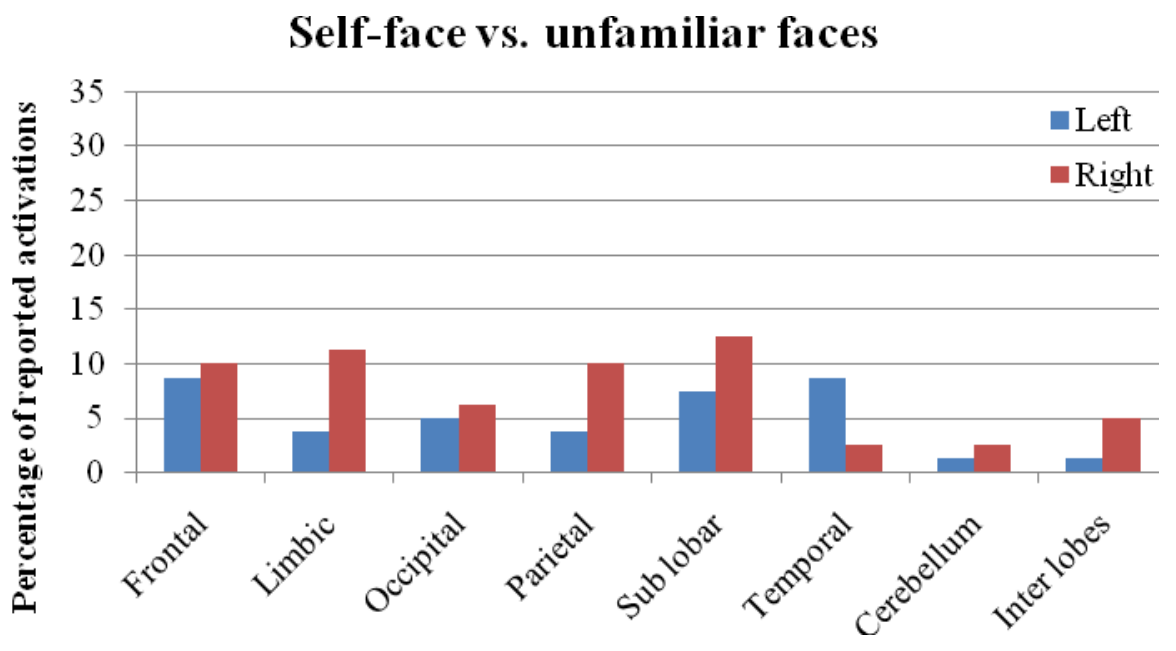

Figure 1a confirms the view obtained from the examination of behavioral performance in the previous section: both hemispheres seem to be implied during self-face recognition, with an advantage for the right one, particularly in the frontal and in the parietal lobes. Figure $1 \mathrm{~b}$ indicates that the self-referential aspects of self-face processing seem to involve the right hemisphere even more preferentially because the contribution of the frontal and parietal lobe is then much more lateralized than when the self-face is compared with unfamiliar faces (see Figure 1c). Table 2 shows the peaks of activations reported in previous studies and gives a more precise idea of the specific regions involved during self-face processing by comparison with that of familiar and unfamiliar faces. Hereafter, we try to sketch the respective role of the regions implied during selfrecognition. We adopted the criterion used by Platek, Wathne, Tierney and Thomson (2008) in discussing only regions activated in five or more studies in total.

Table 2. Number of cerebral areas reported in neuroimaging studies of self-face recognition as a function of the type of contrast used (self-face vs. familiar faces and self-face vs. unfamiliar faces).

\section{LEFT RIGHT TOTAL REFERENCES}

\begin{tabular}{cccc} 
Self-face vs. familiar faces & 8 & 33 & 41 \\
\hline Frontal & 1 & 14 & 15 \\
Inferior frontal gyrus & 0 & 5 & 5
\end{tabular}

$\begin{array}{lllll}\text { Mid-inferior frontal gyrus } & 0 & 1 & 1 & \text { Sugiura et al. (2006) } \\ \text { Middle frontal gyrus } & 0 & 2 & 2 & \text { Platek et al. (2004), (2006) } \\ \text { Medial frontal gyrus } & 1 & 1 & 2 & \text { Platek \& Kemp (2009); Platek et al. (2006) } \\ \text { Superior frontal gyrus } & 0 & 3 & 3 & \begin{array}{l}\text { Platek et al. (2004), (2006); Platek \& Kemp } \\ \text { (2009) }\end{array}\end{array}$

Precentral sulcus $\quad 0 \quad 2 \quad 2 \quad$ Sugiura et al. (2006), (2008)

\begin{tabular}{lllll} 
Inter lobes & $\mathbf{0}$ & $\mathbf{1}$ & $\mathbf{1}$ & \\
Occipito-parietal junction & 0 & 1 & 1 & Sugiura et al. (2006) \\
\hline Limbic & $\mathbf{1}$ & $\mathbf{3}$ & $\mathbf{4}$ & \\
Anterior cingulate cortex & 1 & 1 & 2 & Devue et al. (2007); Platek \& Kemp (2009) \\
Anterior cingulate/paracingulate & $\mathbf{0}$ & 1 & 1 & Platek \& Kemp (2009) \\
Parahippocampal gyrus & 0 & 1 & 1 & Platek \& Kemp (2009) \\
Occipital & $\mathbf{0}$ & $\mathbf{2}$ & $\mathbf{2}$ & \\
Inferior occipital gyrus & 0 & 2 & 2 & Kaplan et al. (2008); Uddin et al. (2005a)
\end{tabular}




\section{Parietal}

Intraparietal sulcus

Inferior parietal lobule

Superior parietal

Posterior superior parietal lobule

Precuneus

Supramarginal gyrus

$\begin{array}{llc}\mathbf{3} & \mathbf{8} & \mathbf{1 1} \\ \mathbf{0} & 1 & 1 \\ 0 & 3 & 3\end{array}$

2

1

1

3

Sugiura et al. (2006)

Kaplan et al. (2008); Platek et al. (2006); Uddin et al. (2005a)

Platek \& Kemp (2009); Uddin et al. (2005a)

Sugiura et al. (2006)

Platek \& Kemp (2009)

Platek \& Kemp (2009); Sugiura et al. (2006), (2008)

\begin{tabular}{lllll}
\hline Sub-lobar & $\mathbf{0}$ & $\mathbf{2}$ & $\mathbf{2}$ & \\
Anterior insula & 0 & 1 & 1 & Devue et al. (2007) \\
Insula & 0 & 1 & 1 & Devue et al. (2007) \\
\hline Temporal & $\mathbf{3}$ & $\mathbf{3}$ & $\mathbf{6}$ & \\
Fusiform gyrus/Inferior temporal gyrus & 1 & 1 & 2 & Sugiura et al. (2006) \\
Inferior temporal gyrus & 1 & 1 & 2 & Sugiura et al. (2008) \\
Middle temporal gyrus & 1 & 0 & 1 & Platek et al. (2006) \\
Superior temporal gyrus & 0 & 1 & 1 & Platek \& Kemp (2009) \\
\hline
\end{tabular}

\begin{tabular}{l} 
Self-face vs. unfamiliar faces \\
\hline Cerebellum \\
Cerebellum \\
\hline
\end{tabular}

\section{Frontal}

Frontal operculum

Inferior frontal gyrus

Inferior frontal gyrus/DLPFC

Middle frontal gyrus

Medial frontal gyrus

Precentral gyrus

\section{Inter lobes}

Occipito-temporo-parietal junction

Post.cingulate cortex/parahippocampal gyrus

Mid-inferior frontal gyrus/Insula

\section{Limbic}

Anterior cingulate cortex

Anterior cingulate gyrus

Anterior cingulate sulcus

Cingulate cortex

Hippocampal formation

\section{Occipital}

Anterior occipital cortex

Inferior occipital gyrus

Occipital cortex

Posterior occipital cortex

Cuneus

Lingual gyrus

$\begin{array}{ccc}32 & 48 & 80 \\ 1 & 2 & 3 \\ 1 & 2 & 3\end{array}$

Kircher et al. (2000), (2001); Platek et al. (2006)

15

Sugiura et al. (2005)

Kircher et al. (2000), Sugiura et al. (2000)

Kircher et al. (2001)

Kircher et al. (2000), (2001); Platek \& Kemp (2009); Sugiura et al. (2000); Taylor et al. (2009)

Platek \& Kemp (2009); Sugiura et al. (2000);

Taylor et al. (2009)

Morita et al. (2008)

\begin{tabular}{lll}
0 & 1 \\
\hline $\mathbf{1}$ & $\mathbf{4}$ & $\mathbf{5}$ \\
0 & 2 & 2 \\
1 & 1 & \\
0 & 1 & $\mathbf{1 2}$ \\
$\mathbf{3}$ & $\mathbf{9}$ & \\
1 & 4 &
\end{tabular}

1

2

2

Morita et al. (2008); Sugiura et al. (2005)

$2 \quad$ Sugiura et al. (2005)

$1 \quad$ Morita et al. (2008)

12

$5 \quad$ Kircher et al. (2000), (2001); Morita et al. (2008);

Taylor et al. (2009)

$2 \quad$ Sugiura et al. (2000)

$\begin{array}{llll}1 & 1 & 2 & \text { Sugiura et al. (2000) } \\ 0 & 1 & 1 & \text { Sugiura et al. (2000) }\end{array}$

$1 \quad 1 \quad 2 \quad$ Taylor et al. (2009)

$02 \quad 2 \quad$ Kircher et al. (2000), (2001)

$\begin{array}{lll}\mathbf{4} & \mathbf{5} & \mathbf{9} \\ 0 & 1 & 1 \\ 1 & 1 & 2 \\ 1 & 0 & 1 \\ 0 & 1 & 1 \\ 1 & 1 & 2 \\ 1 & 1 & 2 \\ \mathbf{3} & \mathbf{8} & \mathbf{1 1}\end{array}$

Morita et al. (2008)

Taylor et al. (2009)

Morita et al. (2008)

Morita et al. (2008)

Taylor et al. (2009)

Taylor et al. (2009) 


\begin{tabular}{|c|c|c|c|c|}
\hline Postcentral gyrus & 0 & 1 & 1 & Platek et al. (2006) \\
\hline Precuneus & 1 & 3 & 4 & $\begin{array}{l}\text { Kircher et al. (2000), (2001); Sugiura et al. } \\
\text { (2000); Taylor et al. (2009) }\end{array}$ \\
\hline Superior parietal & 0 & 1 & 1 & Sugiura et al. (2000) \\
\hline Supramarginal gyrus & 0 & 3 & 3 & $\begin{array}{l}\text { Platek et al. (2006); Platek \& Kemp (2009); } \\
\text { Sugiura et al. (2000) }\end{array}$ \\
\hline Supramarginal gyrus/Inferior parietal lobe & 2 & 0 & 2 & Kircher et al. (2000), (2001) \\
\hline Sub-lobar & 6 & 10 & 16 & \\
\hline Anterior insula & 1 & 0 & 1 & Sugiura et al. (2000) \\
\hline Insula & 1 & 0 & 1 & Morita et al. (2008) \\
\hline Anterior and mid-posterior insula & 0 & 2 & 2 & Kircher et al. (2000), (2001) \\
\hline Caudate nucleus & 1 & 0 & 1 & Sugiura et al. (2000) \\
\hline Globus pallidus & 0 & 1 & 1 & Sugiura et al. (2000) \\
\hline Hypothalamus & 0 & 1 & 1 & Sugiura et al. (2000) \\
\hline Lenticular/subthalamic nucleus & 0 & 2 & 2 & Kircher et al. (2000), (2001) \\
\hline Lentiform nucleus & 1 & 1 & 2 & Platek et al. (2006) \\
\hline Midbrain & 0 & 1 & 1 & Sugiura et al. (2005) \\
\hline Pulvinar & 1 & 1 & 2 & Sugiura et al. (2000) \\
\hline Putamen & 1 & 0 & 1 & Sugiura et al. (2000) \\
\hline Thalamus & 0 & 1 & 1 & Platek et al. (2006) \\
\hline Temporal & 7 & 2 & 9 & \\
\hline Fusiform gyrus & 5 & 1 & 6 & $\begin{array}{l}\text { Kircher et al. (2000), (2001); Sugiura et al. } \\
\text { (2000), (2005); Taylor et al. (2009) }\end{array}$ \\
\hline Superior temporal gyrus & 2 & 1 & 3 & Kircher et al. (2000), (2001); Platek et al. (2006) \\
\hline
\end{tabular}

\section{Prefrontal cortex.}

Inferior frontal gyrus. Activation of the inferior frontal cortex have been reported in six studies comparing the processing of the self-face to that of a familiar face (Devue, Collette, Balteau, Deguedre, Luxen, Maquet et al., 2007; Kaplan, Aziz-Zadeh, Uddin, \& Iacoboni, 2008; Platek, Keenan, Gallup, \& Mohamed, 2004; Sugiura, Sassa, Jeong, Miura, Akitsuki, Horie et al., 2006; Sugiura et al., 2008; Uddin, Kaplan, Molnar-Szakacs, Zaidel, \& Iacoboni, 2005a), all on the right side, and in three studies comparing the processing of the self-face to that of unfamiliar faces, one on the right side (Sugiura, Kawashima, Nakamura, Okada, Kato, Nakamura et al., 2000) and two on the left side (Kircher, Senior, Phillips, Benson, Bullmore, Brammer et al., 2000, Kircher, Senior, Phillips, Rabe-Hesketh, Benson, Bullmore, et al., 2001). In one study, the processing of the self-body by comparison with that of a familiar body also elicited activity in the inferior frontal gyrus, but on the left side (Devue et al., 2007). The right inferior frontal cortex has been hypothetised to be implied in self-other differentiation (Devue et al., 2007; Uddin et al., 2005a) and in the attention to the representation of one's own face (Sugiura et al., 2000). For instance, this region reacts to morphed faces that contain more "self" (Uddin et al., 2005a) and to an intact picture of the self-face during a task requiring to discriminate intact from altered pictures of the self-face and another highly familiar face (Devue et al., 2007). A study presenting participants with pictures of their own face but also with recordings of their own voice showed activity in the inferior frontal gyrus with the two kinds of stimuli suggesting that this structure processes self-referential stimuli of different modalities and that it could contribute to an abstract representation of the self (Kaplan et al., 2008). Recent studies also indicate that this structure might be involved in evaluative judgments about the own face (Kita, Gunji, Sakihara, Inagaki, 
Kaga, Nakagawa et al., 2010; Morita, Itakura, Saito, Nakashita, Harada, Kochiyama et al., 2008; see also the discussion of the present paper).

Medial and middle frontal gyrus. Two studies comparing self-face processing to a familiar face processing found activity in the medial frontal gyrus, one on the right (Platek, Loughead, Gur, Busch, Ruparel, Phend et al., 2006) and one on the left (Platek \& Kemp, 2009). With unfamiliar control faces, two studies reported activity in the right (Platek \& Kemp, 2009; Sugiura et al., 2000) and one bilateral activity (Taylor, Arsalidou, Bayless, Morris, Evans, \& Barbeau, 2009) in the medial frontal gyrus. In a study comparing recognition of the self to recognition of personally familiar faces related (i.e. sibling) or not to the self (i.e. friend), Platek and Kemp (2009) suggested that this region is implied, along with the anterior cingulate gyrus, in complex forms of distinction between self and other (e.g. kinship) and related decisions towards the stimuli (e.g. cooperation vs. aversion).

The middle frontal gyrus was activated on the right side in two studies contrasting self-face and familiar faces (Platek et al., 2004, 2006). When using unfamiliar control faces, three studies reported activity on the left side (Kircher et al., 2000, 2001; Taylor et al., 2009), one on the right side (Platek \& Kemp, 2009) and one bilaterally (Sugiura et al., 2000). In their recent meta-analysis of self-face recognition Platek et al. (2008) asked the question of the origin of the activity in this region which is also often associated with the empathic processing of pain, whereas most selfrecognition studies used neutral faces. They suggest that "neutral faces may possess a level of affective information that is not yet understood" and that activity reported in "left and/or right middle frontal gyrus (...) may be a consequence of variations in affective nature of the respective neutral faces" (p. 179). Recent studies introducing tasks that involve more evaluative and social aspects besides the classical perceptual task of distinguishing between self and other might help answering that question. Platek, Krill and Wilson (2009) showed that activity in the ventral inferior, middle and medial frontal gyri was related to trustworthiness ratings of faces resembling oneself. Moreover, a study by Sui and Han (2007) involving Chinese participants showed that activity in the right middle frontal gyrus increased when they viewed their own face after being primed with an independent pronoun (e.g. "I", "mine") compared to when they were primed with an interdependent pronoun (e.g. "we", "ours"). The authors suggest that stressing the independence of oneself increases the self-other distinction. However, the study by Sugiura et al. (2008) in which they presented their participants with faces and names suggests that the activity in the medial cortical structures is not relevant to distinguish self from others during face or name recognition. These regions thus seem to play a role in the distinction between self and other on a level involving evaluation and where the self is embedded within a social context rather than on a simpler perceptual level.

\section{Insula.}

Four studies comparing the processing of the self-face to that of unfamiliar faces reported activity in the insula, mainly of its anterior part, two on the right side (Kircher et al., 2000, 2001) and two on the left side (Morita et al., 2008; Sugiura et al., 2000). Morita et al. (2008) suggest that activity in the insula reflects an automatic arousal resulting from self-face recognition. Our study using familiar controls also found an implication of the right anterior insula (Devue et al., 2007) during self-face as well as during self-body processing and when collapsing data from self-face and self-body processing. This leads to the idea that this region might be involved in an integrative self-processing, that is, independent of the stimuli used. This idea is supported by the study by Kircher et al. (2000) that showed common activation in the right insula with the self-face and selfdescriptive adjectives. In line with this hypothesis, Platek, Keenan, and Mohamed (2005) suggested that the insula plays a role in making decisions about self-referential information. 
Indeed, the insula has also been found to be implicated in different aspects of self-processing such as self-agency (for a recent review, see Karnath \& Baier, 2010) or autobiographical episodic memory retrieval (Fink et al., 1996).

\section{Cingulate cortex.}

Similarly, the cingulate gyrus has been suggested to be involved during abstract selfprocessing (Devue et al., 2007; Northoff \& Bermpohl, 2004). Involvement of different parts of the cingulate cortex have been consistently reported during self-face recognition by comparison with familiar faces recognition, namely, the anterior part in the right (Devue et al., 2007; Platek \& Kemp, 2009) and a part extending to the paracingulate in the left (Platek \& Kemp, 2009) hemisphere. Studies comparing the self-face to unfamiliar faces also found implication of the right (Kircher et al., 2000, 2001; Morita et al., 2008) or bilateral (Sugiura et al., 2000; Taylor et al., 2009) cingulate cortex, again mainly of its anterior part.

As with the insula, our study involving self-face and self-body processing showed activity in the right anterior cingulate with each kind of stimuli but also when data from the two types of stimuli were collapsed (Devue et al., 2007). This region thus also seems to play a role in the integration of information about oneself independently of the stimulus domain. This is in agreement with the claim that this structure might be generally involved during abstract selfprocessing (i.e., independent of the stimulus domain or of the sensorial modality) or when making decisions about self-referential information (Northoff \& Bermpohl, 2004; Platek et al., 2005).

\section{Temporal cortex.}

Fusiform gyrus and inferior temporal gyrus. Activations of the fusiform gyrus have mainly been reported in the left hemisphere and when comparing the processing of the self-face to that of unfamiliar faces (Kircher et al., 2000, 2001; Sugiura, Watanabe, Maeda, Matsue, Fukuda, \& Kawashima, 2005; Sugiura et al., 2000) but in one case, activity was bilateral (Taylor et al., 2009). When the self-face was compared to familiar faces, but also when stimuli representing the selfbody were used during a familiarity judgment task, the activity in the fusiform gyrus extended to the inferior part of the temporal gyrus, bilaterally (Sugiura et al., 2006). Implication of the bilateral inferior temporal gyrus was also found in another study using a familiarity judgment task (Sugiura et al., 2008). In sum, these findings indicate that the fusiform gyrus and the inferior temporal gyrus would not differentiate the self-face from other faces in terms of self properties but rather that they would allow a first access to the familiarity of a face. Increased activity for the self-face has also been reported in bilateral occipital cortices extending to the inferior temporal gyri and to the fusiform gyrus (Morita et al., 2008) and was interpreted as reflecting a higher attention to the emotional salience of one's own face.

\section{Parietal cortex.}

Inferior parietal lobule. An implication of the right inferior parietal lobule has been reported in three studies contrasting the processing of the self-face to that of a familiar face (Kaplan et al., 2008; Platek et al., 2006; Uddin et al., 2005a). In two studies using unfamiliar faces as control, the activity in the left inferior parietal lobule extended in the supramarginal gyrus (Kircher et al., 2000, 2001). It was suggested that activity in this region might reflect the representation of the self-face as part of a more general awareness of the self-body (Platek et al., 2006; Uddin et al., 2005a) and a self-other discrimination across different sensorial modalities (Uddin et al., 2005a). A study showing specific activation in the right inferior parietal lobe while viewing one's own body compared to a familiar body supports this idea (Hodzic, Kaas, Muckli, Stirn, \& Singer, 2009). 
Supramarginal gyrus. According to three studies, the supramarginal gyrus is implied during self-face recognition by comparison with familiar faces recognition, on the right (Sugiura et al., 2006, 2008) but also on the left (Platek \& Kemp, 2009). An implication of this area has also been found when contrasting the self-face with unfamiliar faces, on the right (Platek \& Kemp, 2009; Platek et al., 2006; Sugiura et al., 2000) and on the left (Kircher et al., 2000, 2001). Based on evidence that this region is damaged in patients showing asomatognosia, some authors have suggested that this region is involved in representing the own face as part of the own body (Sugiura et al., 2000; see also Platek et al., 2006).

Precuneus. The precuneus was activated in four studies comparing the self-face processing to that of unfamiliar faces, in three cases on the right (Kircher et al., 2000, 2001; Sugiura et al., 2000) and in one on the left (Taylor et al., 2009). One study using familiar control faces also reported activity in the left precuneus (Platek \& Kemp, 2009). As noted in two recent metaanalyses (Northoff, Heinzel, de Greck, Bermpohl, Dobrowolny, \& Panksepp, 2006; Platek et al., 2008), this region reacts to different kinds of self-referential stimuli and might be devoted to the integration of different types of self-processing (see also Uddin, Iacobini, Lange, \& Keenan, 2007).

\section{Discussion}

\section{Summary and functions of the self-related areas}

Results from behavioral studies, examination of split-brain patients, TMS and neuroimaging studies point out to a high implication of the right hemisphere but also show clear evidence of an implication of both hemispheres during self-recognition (see Figure 1b), consistent with the idea of a bilateral network for self-recognition (Kircher et al., 2001; Sugiura et al., 2005).

Searching for the cerebral areas involved during self-recognition from neuroimaging studies, some areas seem to emerge consistently. For instance, there is an extensive implication of the prefrontal and parietal cortices (see also Uddin et al., 2005a, 2007). More specifically, within the prefrontal cortex, the inferior frontal gyrus, the medial and middle frontal gyri are often implicated, mostly on the right side. Within the parietal cortex, there is an involvement of the inferior parietal lobule, the supramarginal gyrus and the precuneus, also mainly in the right hemisphere. In addition, other regions such as the anterior cingulate cortex (mainly on the right), the bilateral insula, the fusiform gyrus (mainly on the left), and the bilateral inferior temporal gyrus seem highly involved during self-recognition. To date, the definition of the role of each specific region remains highly hypothetical. Indeed, so far the processing of the self-face has been compared to that of very different "other" faces (from unfamiliar to highly familiar faces) and by means of a variety of tasks. Each of these tasks probably involved a variety of processes, going from a perceptual analysis of facial features allowing face recognition to more unpredictable subjective evaluations and emotions triggered by the vision of the face. Nonetheless, hereafter we will attempt to provide a synthesis of the functions of the neural correlates of self-recognition as proposed by reviewed studies.

The activations in the most posterior parts of the brain, such as in the occipital cortices (Kaplan et al., 2008; Uddin et al., 2005a) presumably reflect perceptual processing. A recent ERP study has also shown that the self-face can already be differentiated from other familiar or unfamiliar faces on the N170 component over occipital regions (Keyes, Brady, Reilly, \& Foxe, 2010). This early component is supposed to reflect structural encoding of faces. This occipital activity extends to the inferior temporal gyri and the fusiform gyrus (e.g. Morita et al., 2008) which might result from a first differentiation between faces of different levels of familiarity (Devue et al., 2007) before the identification of the faces. Indeed, activity in the left fusiform gyrus was mainly found when comparing the self-face to unfamiliar faces (Kircher et al., 2000, 
2001; Sugiura et al., 2000, 2005; Taylor et al., 2009). One might wonder why perceptual analysis of one's own face would differ from that of other faces. These self-specific posterior activations might result from the fact that we do not process the same kind of structural information when seeing our own face and other faces because processing goals are different in each case. The aim of processing another person's face is typically to identify that person or to interpret her emotional expressions whereas the goal of processing our own face is normally not identification but an inspection of facial features, for instance when grooming (Greenberg \& Goshen-Gottstein, 2009; Keyes \& Brady, 2010)

Regions of the parietal cortex such as the inferior parietal lobule and the supramarginal gyrus have often been attributed a role in terms of spatial representation of oneself (e.g. Platek et al., 2006; Sugiura et al., 2000; Uddin et al., 2005a). The supramarginal gyrus, along with the occipito-parietal junction might be part of an occipito-parietal network (dorsal pathway) implied in a visuo-spatial representation of oneself, in other words, with the self being represented as an object in the space and with the self-face being represented as a part of the own body (Platek et al., 2006; Sugiura et al., 2000; Uddin et al., 2005a).

There would be direct connections between the parietal and the frontal cortices during selfrecognition. In their recent review about the self and social cognition, Uddin et al. (2007) suggested that right frontoparietal areas activated during self-recognition overlap with the mirror neuron system, comprising the inferior frontal cortex and the inferior parietal lobule. They posit that this network is involved in representing different aspects of the physical self, and in relating the physical self to others through motor simulation mechanisms. During self-recognition, one would process the perceived physical self (e.g. the self-face) using a similar mechanism. The perceived self would be mapped onto the perceiver's own motor repertoire. Uddin et al. (2007) suggested that a second network involving the midline cortical structures and overlapping with some areas of a "default-mode" network including the ventral and dorsal prefrontal cortex, the precuneus and posterior lateral cortices would play a role in more abstract self- and other-related processing, in terms of mental states attribution and evaluation.

Activations within the frontal cortex have been attributed to more complex forms of selfother differentiation and self-evaluation and this region would be of particular importance for social cognition. For instance, the right inferior frontal gyrus would contribute to complex perceptual judgments in terms of comparison to an "ideal" self (Morita et al., 2008). Its activation might also correspond to the sustained attention to one's own face (Sugiura et al., 2000) which might itself allow or reflect evaluative processes (Kita et al., 2010; Morita et al., 2008; see also Heinisch et al., 2010). Accordingly, ERP studies indicate that the self-face is a particularly salient stimulus because it elicits ampler P300 than other faces (Miyakoshi, Nomura, \& Ohira, 2007; Ninomiya, Onitsuka, Chen, Sato, \& Tashiro, 1998). Similarly the medial frontal gyrus would allow the detection of self-resemblance in terms of kinship along with the cingulate cortex (Platek \& Kemp, 2009) or during judgment of trustworthiness along with the ventral inferior and with the middle frontal gyri (Platek et al., 2009; but see Verosky \& Todorov, 2010). Various regions within the frontal cortex as well as the insula and the cingulate cortex have also been hypothesized to play a role in the construction of an abstract representation of oneself (i.e. a sense of "me"), independently of the type of stimulus presented or of the sensorial modality stimulated (see e.g. Devue et al., 2007; Kaplan et al., 2008; Kircher et al., 2000, 2001). According to Uddin et al. (2007), because it is part of the cortical midline structures network and because it has direct connections with the posterior component of the mirror neurons system (i.e. the inferior parietal lobule), the precuneus would allow interactions between these two networks and would be of high importance in the elaboration of integrated information about oneself. 
There are thus at least four candidate regions for processing self-related stimuli at a higher, integrated level, namely, the inferior frontal gyrus (Kaplan et al., 2008), the anterior insula (Devue et al., 2007; Kircher et al., 2000), the anterior cingulate cortex (Devue et al., 2007; Northoff \& Bermpohl, 2004; Platek et al., 2005) and the precuneus (Northoff et al., 2006; Platek et al., 2008; Uddin et al., 2007). However, this idea of integration might be confounded with other kinds of responses that could be common to different self-related material, such as evaluative judgments or emotional reactions. The notion of general "decision making about oneself" used by some authors could correspond to such responses. For instance, the task used in the Devue et al. (2007) study involved detection of alterations affecting the faces (interocular distance) or the bodies (waist to hip ratio) of the participant herself or of her colleague. We hypothesized that activity found in the insula and the cingulate cortex reflected an abstract representation of oneself but it might actually originate from subjective judgments (e.g. evaluation) or a subsequent emotional reaction in response to one's own pictures. The same can be said from inferior frontal gyrus activation following the presentation of the own face and of the own voice in the study by Kaplan et al. (2008). This activity might reflect some subjective judgments emitted by people when seeing their own face (e.g. "I look tired on that picture") and when hearing their own voice (e.g. "I sound funny"), judgments that might be followed by some emotional reactions (e.g. feeling ashamed), rather than an abstract integration of information. As a consequence, the exact role of these "higher-level" regions still needs to be clarified.

When considering self processing in more general terms, Northoff and Bermpohl (2004) suggested that integration processes would take place in the posterior cingulate whereas evaluative processes would be subtended by the dorsolateral prefrontal cortex. Regarding self-recognition, the distinction between the integration of the physical aspects of the self and the evaluative judgments about these physical aspects is very difficult. Indeed, it is likely that even if the task used only requires a perceptual judgment, participants also automatically emit specific judgments about themselves which in turn triggers activity linked to these processes. Researchers in this field probably all know that participants in their study are almost never indifferent about the way they look on the pictures (see e.g. Kita et al., 2010). The next step in understanding the role of selfspecific regions is thus to take more subjective and emotional variables and the variety of the cognitive processes involved when looking at one's own face into account. We will now consider the more specific issue of the relationship between self-face recognition and self-awareness.

\section{Relation between self-recognition and self-awareness.}

Does self-face recognition equal self-awareness? There is no consensual answer to that question. For Keenan and his colleagues self-awareness includes knowing that one is a separate entity. Self-awareness also involves being aware of one's thoughts, or mental state, and encompasses the ability to imagine oneself in the future or in the past (e.g. Keenan et al., 2003a; Keenan, Rubio, Racioppi, Johnson, \& Barnacz, 2005). These researchers argued that self-face recognition appears to be related to high-order awareness and that "the self-face may be an ideal stimulus to begin the investigations of higher-order consciousness and the brain" (e.g. Keenan et al., 2005, p. 697). By contrast, other researchers considered that self-face recognition does not imply such a sophisticated kind of self-awareness. In several papers, Mitchell (1993) and Morin (2002; 2007) admitted that self- recognition requires self-attention and a certain kind of selfknowledge. However, according to these authors, the kind of self-information that is required for recognizing oneself in a mirror is a kinesthesic representation of one's own body. Briefly speaking, the participant (in the context of these papers, participants of interest were usually chimpanzees or infants) matches the representation of the physical self with the reflection seen in the mirror and concludes "it's me" (Morin, 2007, p.1068). This argumentation is relevant as far as 
self-mirror recognition is concerned. In the studies reviewed in the present paper, participants were not placed in front of a mirror but were presented with pictures. Therefore, kinesthesic information was not available. We think that, like self-mirror recognition, self-face recognition from pictures does not require access to one's own mental states or thoughts. According to models of face recognition the intervention of such high-level cognitive processes is not necessary for recognizing a face. Face recognition requires matching the current representation of the seen face's surface structure with a stored representation in the perceptual memory system devoted to faces (Bruce and Young, 1986). After a face has been recognized, identity- specific semantic information about the person may be retrieved. However, the fact that face recognition does not require high-level cognitive processes does not mean that recognizing a face may not be accompanied with such more complex processes. For instance, recent research demonstrated that conscious recollection, which is associated with autonoetic awareness (Tulving, 1985), may accompany the recognition of famous (Damjanovic \& Hanley, 2007) and personally familiar faces (Barsics \& Brédart, 2010). It is therefore highly probable that such conscious recollection also accompanies self-recognition in some circumstances.

Neuroscientific research on self-face recognition has focused mostly on localizing its neural substrates rather than on determining cognitive operations induced by the perception of the self-face (Tsakiris, 2008). At this stage of research, it seems necessary to clarify what we are searching for when we use self-face as stimuli in experiments. We mentioned here above that some researchers consider that the self-face may be an ideal stimulus to investigate the brain correlates of higher-order consciousness (Keenan et al., 2003a; 2005). We think that if a study is aimed at determining which brain regions are selectively involved in complex cognitive operations such as mental travel in the past and the future, or mentalizing, then stimuli and procedures that are specifically designed to tap these functions should be used rather than using a self-face recognition task. A number of works did already resort to such a more targeted strategy for studying the brain correlates of mental travel in the past and future (for a recent review, see Szupnar, 2010) or intention understanding (for a review, see Van Overwalle, 2009).

Although a relationship between self-recognition and self-awareness has been assumed in the literature, it is only recently that neuroimaging studies started to examine whether separate brain regions recruited during self-recognition were specifically associated with behavioral measures of different aspects of self-awareness, for instance, public self-awareness induced by the perception of the self-face $v s$ evaluation of the self-face toward a standard (see Morita et al., 2008). Perceiving the self-face is likely to direct attention on one's own appearance i.e; public self-awareness (Morin, 2006). In turn, focusing on the self may yield self-evaluation, i.e. a comparison of the seen self-face against a mental representation an ideal self-face (for a review see Carver, 2003). If a discrepancy is found between the perceived and the ideal representation of the self-face, then the observer can experience negative emotions or feelings such as embarrassment. Brain correlates of public self-awareness induced in the participant by the perception of her/his own face could be different from those of self-evaluation following selfrecognition. Recently, Morita et al. (2008) reported data supporting this hypothesis by scanning participants whose task was to evaluate how photogenic seen faces of self and friends were. After scanning, participants completed a questionnaire evaluating their public self-consciousness (i.e. their tendency to be aware of their own appearance) as well as a task consisting in rating the intensity of the embarrassment they experienced while seeing each presented face. Results showed that the right precentral gyrus was more strongly activated when participants with high public selfconsciousness viewed their own faces compared with participants with low public selfconsciousness. However, activity in the right precentral gyrus was not correlated with the level of embarrassment experienced by seeing non-photogenic self-pictures. In addition, Morita et al. 
(2008) showed that, conversely, activity in the right middle inferior frontal gyrus was not related with levels of public self-consciousness but was modulated by the extent of embarrassment (the outcome of self-evaluation). These results demonstrated a functional dissociation between two brain correlates of self-recognition: the activation of the right precentral gyrus is associated with public self-awareness while the right middle inferior frontal gyrus is associated with selfevaluation induced by the perception of one's own face.

\section{Conclusion}

During the last ten years a considerable number of studies were aimed at localizing the neural correlates of self-face recognition. The real objective of most of these studies was, in fact, to gain knowledge about self-awareness rather than self-face recognition per se. Self-recognition was considered as an indicator of self-awareness (e.g. Feinberg \& Keenan, 2005; Keenan et al., 2005; Sugiura et al., 2005; Sui et al., 2006; Uddin et al., 2005; for a critical view see Morin, 2002; 2007), the level of such self-awareness being often left unspecified. As shown in the present paper and in other recent reviews (e.g. Platek et al., 2008), researchers discovered many different cerebral areas that are associated with self-face recognition. Unfortunately, it remains an extremely difficult and hazardous task to relate specific cognitive operations induced by selfperception and brain regions recruited. We do not think that understanding the neural correlates of self-face recognition (and some forms of related self-awareness) will significantly improve without specifying more clearly what happens when a participant sees her or his face on the screen, and what is specific to self-recognition in comparison with the recognition of another highly familiar face. Intuitively, the perception of one's own face should trigger the structural representation of the self-face in the perceptual representational system, and allow selfrecognition. This recognition is likely to induce public self-awareness which in turn may yield some kind of self-evaluation. The result of such an evaluation may be accompanied with emotional responses. Similar hypothetical sequences of operations following self-perception have earlier been proposed by others (e.g. Carver, 2003; Morita et al., 2008), however fine-grained empirical evaluation of such proposals is needed. Otherwise, we mentioned earlier that it has been demonstrated that the recognition of familiar faces is prone to produce a retrieval of episodic information (autonoetic awareness). It is highly probable that such memories may accompany self-recognition. We think that one of the major aims of research on the neural correlates of selfrecognition should be to identify more clearly the cognitive components induced by the perception of the self-face, and to relate them with the brain regions that have been shown to be associated with self-face recognition.

\section{References}

Amsterdam, B. (1972). Mirror self-image reactions before age two. Developmental Psychobiology, 5, 297-305.

Barsics, C., \& Brédart, S. (2010). Recalling episodic information about personally known faces and voices. Consciousness and Cognition, in press

Bluhm, C., \& Clendenin, N. (2009). Someone else's face in the mirror: Identity and the new science of face transplants. Westport, CT: Praeger.

Brady, N., Campbell, M., \& Flaherty, M. (2004). My left brain and me: A dissociation in the perception of self and others. Neuropsychologia, 42, 1156-1161.

Breen, N., Caine, D., \& Coltheart, M. (2001). Mirrored-self misidentification: two cases of focal onset dementia. Neurocase, 7, 239-254. 
Bruce, V., \& Young, A. W. (1986). Understanding face recognition. British Journal of Psychology, 77, 305-327.

Carver, C.S. (2003) Self-awareness. In M.R. Leary \& J.P. Tangney (Eds.), Handbook of self and Identity (pp. 179-196). New York: Guilford Press.

Cole, J. (1998). About face. Cambridge, MA: MIT Press.

Damjanovic, L., \& Hanley, J.R. (2007). Recalling episodic and semantic information about famous faces and voices. Memory \& Cognition, 35, 1205-1210.

Devue, C., \& Brédart, S. (2008). Attention to self-referential stimuli: Can I ignore my own face? Acta Psychologica, 128, 290-297.

Devue, C., Collette, F., Balteau, E., Degueldre, C., Luxen, A., Maquet, P., \& Brédart, S. (2007). Here I am: The cortical correlates of visual self-recognition. Brain Research, 1143, 169-182.

Feinberg, T.E., \& Keenan, J.P. (2005). Where in brain is the self. Consciousness and Cognition, 14, 661-678.

Fink, G.R., Markowitsch, H.J., Reinkemeier, M., Bruckbauer, T., Kessler, J., \& Heiss, W-D. (1996). Cerebral representation of one's own past: Neural networks involved in autobiographical memory. Journal of Neuroscience, 16, 4275-4282.

Gallup, G.G. (1970). Chimpanzees: self-recognition. Science, 167, 86-87.

Greenberg, S.N., \& Goshen-Gottstein, Y. (2009)? Not all faces are processed equally: Evidence for featural rather than holistic processing of one's own face in a face-imaging task. Journal of Experimental Psychology: Learning, Memory, and Cognition, 55, 499-508.

Heinisch, C., Dinse, H.R., Tegenthoff, M., Juckel, G., Brüne, M. (2010). An rTMS study into selfface recognition using video-morphing technique. Social Cognitive and Affective Neuroscience, 5, 356-361.

Hodzic, A., Kaas, A., Muckli, L., Stirn, A., \& Singer, W. (2009). Distinct cortical networks for the detection and identification of human body. Neuroimage, 45, 1264-1271.

Hughes, S.M., \& Nicholson, S.E. (in press). The processing of auditory and visual recognition of self-stimuli. Consciousness and Cognition.

Kaplan, J. T., Aziz-Zadeh, L., Uddin, L., \& Iacoboni, M. (2008). The self across the senses: The neural response to one's own face and voice. Social, Cognitive, and Affective Neuroscience, 3, 218-223.

Karnath, H.O., \& Baier, B. (2010). Right insula for our sense of ownership and self-awareness of actions. Brain Structure and Function, 214, 411-417.

Keenan, J.P., Freund, S., Hamilton, R.H., Ganis, G., \& Pascual-Leone, A. (2000). Hand response differences in a self-face identification task. Neuropsychologia, 38, 1047-1053.

Keenan, J.P., Gallup, G.G., \& Falk, D. (2003a). The face in the mirror: The search for the origins of consciousness. New York: HarperCollins/Ecco.

Keenan, J.P., Ganis, G., Freund, S., \& Pascual-Leone, A. (2000). Self-face identification is increased with left hand responses. Laterality, 5, 259-268.

Keenan, J.P., McCutcheon, B., Freund, S., Gallup, G.G., Sanders, G., \& Pascual-Leone, A. (1999). Left hand advantage in a self-face recognition task. Neuropsychologia, 37, 14211425.

Keenan, J.P., Nelson, A., O’Connor, M., \& Pascual-Leone, A. (2001). Self-recognition and the right hemisphere. Nature, 409, 305.

Keenan, J.P., Rubio, J., Racioppi, C., Johnson, A., \& Barnacz, A. (2005). The right hemisphere and the dark side of consciousness. Cortex, 41, 695-704. 
Keenan, J.P., Wheeler, M., Platek, S.M., Lardi, G., \& Lassonde, M. (2003b). Self-face processing in a callosotomy patient. European Journal of Neuroscience, 18, 2391-2395.

Keyes, H., \& Brady, N. (2010). Self-face recognition is characterized by "bilateral gain" and by faster, more accurate performance which persists when faces are inverted. The Quarterly Journal of Experimental Psychology, 63, 840-847.

Keyes, H., Brady, N., Reilly, R.B., \& Foxe, J.J. (2010). My face or yours? Event-related potential correlates of self-face processing. Brain and Cognition, 72, 244-254.

Kircher, T.T.J., Senior, C., Phillips, M.L., Benson, P.J., Bullmore, E.T., Brammer, M., Simmons, A., Williams, S.C.R., Bartels, M., \& David, A.S. (2000). Towards a functional neuroanatomy of self processing: Effects of faces and words. Cognitive Brain Research, 10, 133-144.

Kircher, T.T.J., Senior, C., Phillips, M.L., Rabe-Hesketh, S., Benson, P.J., Bullmore, E.T., Brammer, M., Simmons, A., Bartels, M., \& David, A.S. (2001). Recognizing one's own face. Cognition, 78, B1-B15.

Kita, Y., Gunji, A., Sakihara, K., Inagaki, M., Kaga., M., Nakagawa, E., Hosokawa, T. (2010). Scanning strategies do not modulate face identification: Eye-tracking and near-infrared spectroscopy study. PLoS ONE, 5, e11050.

Ma, Y., \& Han, S. (2010). Why we respond faster to the self than to others? An implicit positive association theory of self-advantage during implicit face recognition. Journal of Experimental Psychology: Human Perception and Performance, 36, 619-633.

McNeill, D. (1998). The face. Boston: Little, Brown and Company.

Mitchell, R.W. (1993). Mental models of mirror self-recognition: Two theories. New Ideas in Psychology, 11, 295-325.

Miyakoshi, M., Kanayama, N., Iidaka, T., \& Ohira, H. (2010). EEG evidence for face-specific visual self-representation. NeuroImage, 50, 1666-1675.

Miyakoshi, M., Nomura, M., \& Ohira, H. (2007). An ERP study on self-relevant object recognition. Brain and Cognition, 63, 182-189.

Morin, A. (2002). Right hemispheric self-awareness : A critical assessment. Consciousness and Cognition, 11, 396-401.

Morin, A. (2006). Levels of consciousness and self-awareness: A comparison and integration of various neurocognitive view. Consciousness and Cognition, 15, 358-371.

Morin, A. (2007). Self-awareness and the left hemisphere: The dark side of selectively reviewing the literature. Cortex, 43, 1068-1073.

Morita, T., Itakura, S., Saito, D.N., Nakashita, S., Harada, T., Kochiyama ,T., \& Sadato, N. (2008). The role of the right prefrontal cortex in self-evaluation of the face: A functional magnetic resonance imaging study. Journal of Cognitive Neuroscience, 20, 342-355.

Ninomiya, H., Onitsuka, T., Chen, C.H., Sato, E., \& Tashiro, N. (1998). P300 in response to the subject's own face. Psychiatry and Clinical Neurosciences, 52, 519-522.

Northoff, G., \& Bermpohl, F. (2004). Cortical midline structures and the self. Trends in Cognitive Sciences, 8, 102-107.

Northoff, G., Heinzel, A., de Greck, M., Bermpohl, F., Dobrowolny, H., \& Panksepp, J. (2006). Self-referential processing in our brain-a meta-analysis of imaging studies on the self. Neuroimage, 31, 440-457.

Platek, S.M., \& Gallup, G.G. (2002). Self-face recognition is affected by schizotypal personality traits. Schizophrenia Research, 57, 81-85.

Platek, S.M., Keenan, J.P., Gallup, G.G.Jr., \& Mohamed, F.B. (2004). Where am I? The neurological correlates of self and other. Cognitive Brain Research, 19, 114-122. 
Platek, S.M., Keenan, J.P., Mohamed, F.B. (2005). Sex differences in the neural correlates of child facial resemblance: an event related fMRI study. NeuroImage, 25, 1336-1344.

Platek, S.M., \& Kemp, S.M. (2009). Is family special to the brain? An event-related fMRI study of familiar, familial, and self-face recognition. Neuropsychologia, 47, 849-858.

Platek, S.M., Krill, A.L., \& Wilson, B. (2009). Implicit trustworthiness ratings of self-resembling faces activate brain centers involved in reward. Neuropsychologia; 47, 289-293.

Platek, S.M., Loughead, J.W., Gur, R.C., Busch, S., Ruparel, K., Phend, N., Panyavin, I.S., \& Langleben, D.D. (2006). Neural substrates for functionally discriminating self-face from personally familiar faces. Human Brain Mapping, 27, 91-98.

Platek, S.M., Thomson, J.W., \& Gallup, G.G. (2004). Cross-modal self-recognition: The role of visual, auditory, and olfactory primes. Consciousness and Cognition, 13, 197-210.

Platek, S.M., Wathne, K., Tierney N.G., \& Thomson, J.W. (2008). Neural correlates of self-face recognition: An effect-location meta-analysis. Brain Research, 1232, 173-184.

Sperry, R.W., Zaidel, E., \& Zaidel, D. (1979). Self-recognition and social awareness in the disconnected minor hemisphere. Neuropsychologia, 17, 153-166.

Sugiura, M., Kawashima, R., Nakamura, K., Okada, K., Kato, T., Nakamura, A., Hatano, K., Itoh, K., Kojima, S., \& Fukuda, H. (2000). Passive and active recognition of one's own face. NeuroImage, 11, 36-48.

Sugiura, M., Sassa, Y., Jeong, H., Horie, K., Sato, S., \& Kawashima, R. (2008). Face-specific and domain-general characteristics of cortical responses during self-recognition. NeuroImage, 42, 414-422.

Sugiura, M., Sassa, Y., Jeong, H., Miura, N., Akitsuki, Y., Horie, K., Sato, S., \& Kawashima, R. (2006). Multiple brain networs for visual self-recognition with different sensitivity for motion and body part. NeuroImage, 32, 1905-1917.

Sugiura, M., Watanabe, J., Maeda, Y., Matsue, Y., Fukuda, H., \& Kawashima, R. (2005). Cortical mechanisms of visual self-recognition. NeuroImage, 24, 143-149.

Sui, J., \& Han, S. (2007). Self-construal priming modulates neural substrates of self-awareness. Psychological Science, 18, 861-866.

Sui, J., Liu, C.H., \& Han, S. (2009). Cultural differences in neural mechanisms of self-recognition. Social Neuroscience, 4, 402-411.

Sui, J., Zhu, Y., \& Han, S. (2006). Self-face recognition in attended and unattended conditions: An event-related brain potential study. Neuroreport, 17, 423-427.

Spuznar, K.K. (2010). Episodic future thought: An emerging concept. Perspectives in Psychological Science, 5, 142-162.

Tacikowski, P., \& Nowicka, A. (2010). Allocation of attention to self-name and self-face: An ERP study. Biological Psychology, 84, 318-324.

Taylor, M.J., Arsalidou, M., Bayless, S.J., Morris, D., Evans J.W., \& Barbeau, E.J. (2009). Neural correlates of personally familiar faces: Parents, partner and own faces. Human Brain Mapping, 30, 2008-2020.

Tsakiris, M. (2008). Looking for myself: Current multisensory imput alerts self-face recognition. PLOS ONE, 3, e4040.

Tulving, E. (1985). Memory and consciousness. Canadian Psychology, 26, 1-12.

Turk, D.J., Heartherton, T.F., Kelley, W.M., Funnell, M.G., Gazzaniga, M.S., \& Macrae, C.N. (2002). Mike or me? Self-recognition in a split-brain patient. Nature Neuroscience, 5, 841842.

Uddin, L.Q., Iacobini, M., Lange, C., \& Keenan, J.P. (2007). The self and social cognition: the role of cortical midline structures and mirror neurons. Trends in Cognitive Sciences, 11, 153-157. 
Uddin, L.Q., Kaplan, J.T., Molnar-Szakacs, I., Zaidel, E., \& Iacoboni, M. (2005a). Self-face recognition activates a frontoparietal "mirror" network in the right hemisphere: An eventrelated fMRI study. NeuroImage, 25, 926-935.

Uddin, L.Q., Molnar-Szakacs, I., Zaidel, E., \& Iacobini, M. (2006). rTMS to the right inferior parietal lobule disrupts self-other discrimination. Social Cognitive and Affective Neuroscience, 1, 65-71.

Uddin, L.Q., Rayman, J., \& Zaidel, E. (2005b). Split-brain reveals separate but equal selfrecognition in the two cerebral hemispheres. Consciousness and Cognition, 14, 633-640.

Van Overwalle, F. (2009). Social Cognition and the brain: A meta-analysis. Human Brain Mapping, 30, 829-858.

Verosky, S.C.; \& Todorov, A. (2010). Differential neural responses to faces physically similar to the self as a function of their valence. NeuroImage, 49, 1690-1698. 